\title{
État des lieux sanitaire et bucco-dentaire de la population gériatrique libanaise
}

N. El Osta, ${ }^{7}$ M. Hennequin, ${ }^{2}$ L. El Osta, ${ }^{3}$ N. Bou Abboud Naaman, ${ }^{4}$ N. Geahchan ${ }^{3}$ et S. Tubert-Jeannin ${ }^{2}$

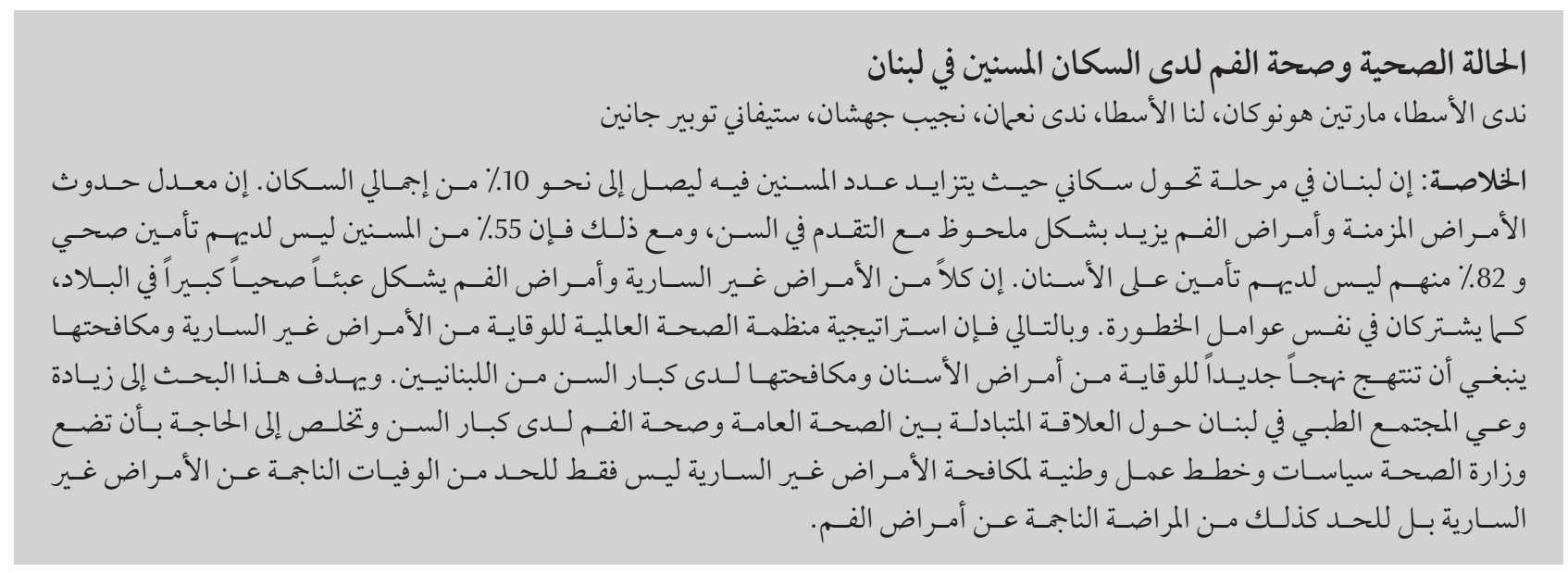

RÉSUMÉ Le Liban est en phase de transition démographique, de plus en plus de personnes atteignant un âge avancé ; le pourcentage de personnes âgées y est de 10 \%. L'incidence des maladies chroniques et buccodentaires augmente considérablement avec l'âge. Or 55 \% des personnes âgées n'ont pas d'assurance maladie et $82 \%$ n'ont pas de couverture dentaire. Les maladies non transmissibles et les maladies bucco-dentaires, qui constituent un important fardeau sanitaire dans le pays, partagent les mêmes facteurs de risque. La stratégie de I'OMS pour la lutte contre les maladies non transmissibles devrait donc constituer une nouvelle façon d'aborder la prévention et le contrôle des maladies bucco-dentaires chez les Libanais âgés. Le présent article vise à sensibiliser la communauté médicale au Liban à la corrélation entre santé générale et santé bucco-dentaire chez les personnes âgées et conclut par la nécessité pour le ministère de la Santé d'élaborer des politiques et plans d'action nationaux de lutte contre les maladies non transmissibles afin de réduire non seulement la mortalité due à ces dernières mais aussi la morbidité associée aux maladies bucco-dentaires.

\section{Status of health and oral health of the elderly population in Lebanon}

ABSTRACT Lebanon is in demographic transition as more people reach increasingly older age; $10 \%$ of the population in Lebanon is elderly. The incidence of chronic diseases and oral diseases increases significantly with age. However, 55\% of the elderly have no health insurance and $82 \%$ have no dental insurance. Both noncommunicable diseases (NCDs) and oral diseases are a major health burden in the country and share the same risk factors. The WHO strategy for prevention and control of noncommunicable diseases should therefore be a new approach for the prevention and control of dental diseases among Lebanese elderly. This paper aims to increase the awareness of the medical community in Lebanon about the interrelationship between general and oral health in the elderly and concludes with the need for the Ministry of Health to develop policies and national action plans against NCDs to reduce not only mortality from NCDs but also morbidity from oral diseases. 


\section{Vieillissement démographique de la population libanaise}

Le Liban, comme la plupart des pays en développement, est en phase de transition démographique (1). Selon l'Administration centrale des Statistiques, la proportion de Libanais âgés a significativement augmenté au cours des 40 dernières années. Celle-ci, qui était de 4,9\% en 1970 et de $7,5 \%$ en 2004 , a atteint $9,6 \%$ en 2007. Le vieillissement au Liban est la résultante de l'allongement significatif de l'espérance de vie, qui a augmenté d'environ 11 ans au cours des 50 dernières années. En 2010, l'espérance de vie des hommes au Liban était de 70,4 ans et celle des femmes de 74,76 ans. Ce vieillissement est aussi la résultante de la diminution de la natalité. Ainsi, la proportion d'enfants de moins de 4 ans, qui était de $16 \%$ environ en 1970, a significativement diminué en 2007 pour atteindre $7 \%$ (Figure 1). En outre, le nombre d'émigrés a eu un impact sur la composition démographique de la population libanaise par la réduction de la catégorie des jeunes Libanais en âge de travailler, contribuant au vieillissement de la population (1).

\section{Impact du vieillissement sur la santé bucco- dentaire et générale}

Le vieillissement a un impact considérable sur la santé. À mesure que les gens vieillissent, leur susceptibilité aux maladies chroniques, aux maladies bucco-dentaires et aux infections augmente et s'aggrave par des déficits du système immunitaire (2). Le risque carieux et la prévalence de la maladie parodontale augmentent de façon importante, les muqueuses buccales deviennent atrophiques et présentent progressivement un aspect fin et lisse, associé à une perte d'élasticité.Également, les modifications histologiques des glandes salivaires sont responsables de la sécheresse buccale (3).

Les interrelations entre la santé générale et la santé bucco-dentaire sont particulièrement prononcées chez les personnes âgées (4). De nombreuses affections générales accroissent le risque de maladies bucco-dentaires. Ainsi la perturbation de la coordination neuromusculaire, la diminution de la vision, la réduction de la dextérité manuelle et la perte de l'autonomie empêchent la réalisation d'une hygiène buccale ainsi que l'accès aux soins (2). Les maladies bucco-dentaires, à leur tour, peuvent favoriser l'apparition ou l'aggravation de nombreux problèmes de santé. La perte des dents entraîne une diminution du potentiel masticatoire et favorise le changement du type d'alimentation. Les maladies parodontales peuvent décompenser un diabète préexistant. Elles peuvent également augmenter le risque de développement de lésions d'athérosclérose, de cardiopathies ischémiques, d'accidents vasculaires cérébraux ischémiques et d'infections respiratoires (4).

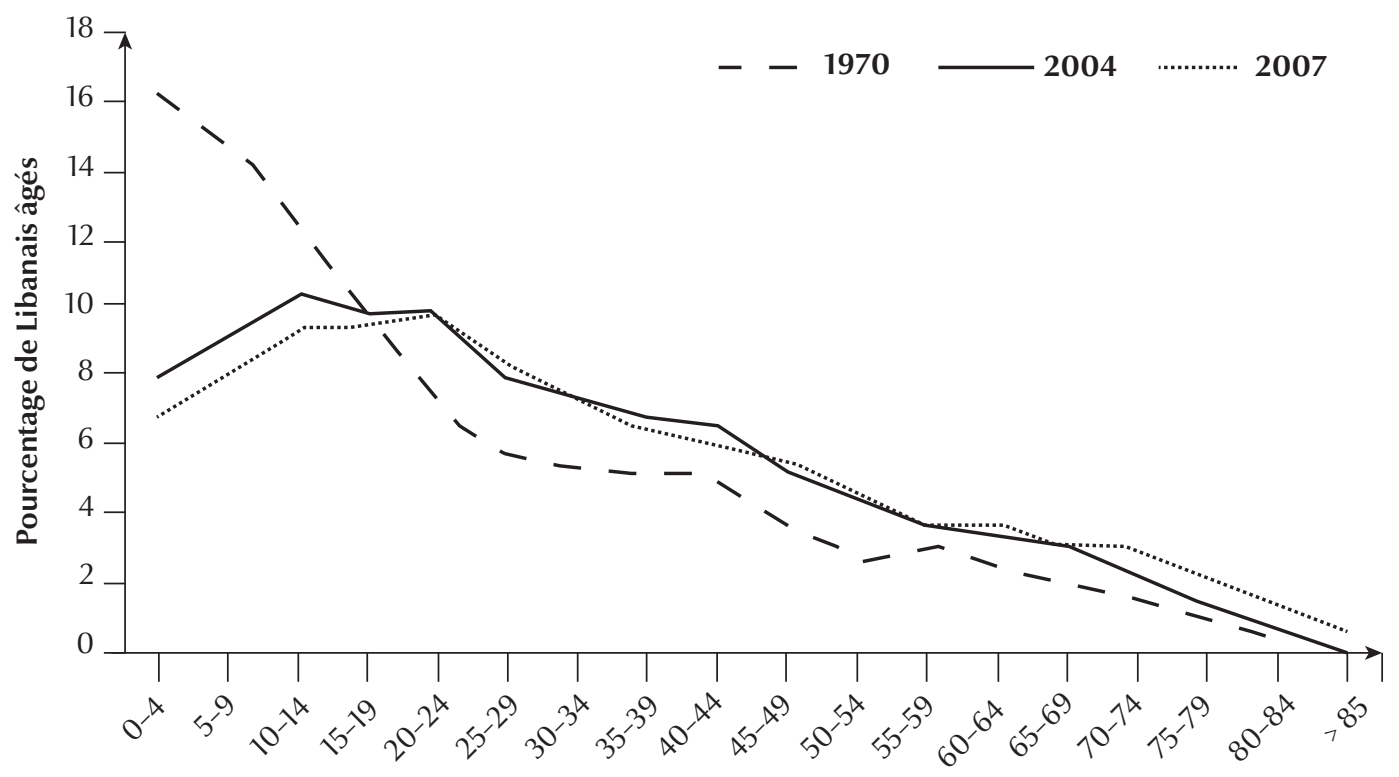

Catégories d'âge (ans) 


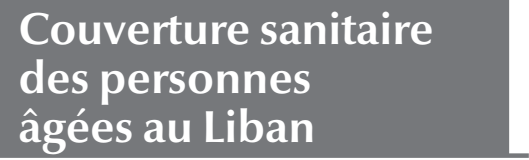

La santé bucco-dentaire est une composante essentielle de la santé générale qui est essentielle au bienêtre, d'où le rôle primordial des gouvernements pour garantir une assurance maladie et dentaire à leurs citoyens (2). Au Liban, les systèmes de protection sociale sont défectueux dans la couverture de santé : le pourcentage de personnes âgées de plus de 65 ans bénéficiant d'une assurance maladie ne dépasse pas $45 \%$, alors que seulement $18 \%$ bénéficient d'une protection bucco-dentaire. Concernant les personnes plus âgées (> 85 ans), les chiffres sont plus alarmants. Seulement $33,2 \%$ de ces personnes disposent d'une protection sanitaire et $20 \%$ d'une couverture dentaire (Tableau 1) (1).

Le système d'assurance maladie au Liban, bien que défaillant, est très diversifié. Ainsi, le ministère de la Santé couvre quelques traitements des maladies du troisième âge (dialyse, chirurgie cardiaque et traitement anticancéreux). La Caisse Nationale de Sécurité Sociale (CNSS) couvre les salariés déclarés et, très marginalement, quelques professions libérales; les salariés sont exclus de la couverture santé dès qu'ils atteignent l'âge de la retraite, au moment où ils en ont le plus besoin. Pour les salariés du secteur public couverts par la Coopérative du secteur public, ainsi que pour les corps militaires couverts par la protection sociale des secteurs non civils, la situation est meilleure : les prestations sont élevées et les salariés ne sont pas exclus après la retraite.

Devant l'insuffisance de la plupart des couvertures sociales et la faiblesse des remboursements, certains Libanais ont recours à l'assurance privée qui, d'une part, coûte cher et d'autre part, n'est pas accessible aux individus souffrant de maladies chroniques préexistantes (1).

À noter que les assurances privées en général, le ministère de la Santé et la CNSS ne couvrent pas les soins de santé bucco-dentaire. Ceux-ci sont seulement pris en charge pour les assurés du secteur militaire et des forces de sécurité et sont incomplètement remboursés par la Coopérative du secteur public. Les soins dentaires dépendent de ce fait des considérations financières inaccessibles pour les sujets âgés de niveau économique principalement bas; dans ce cas de figure, les facteurs socio-économiques peuvent limiter l'accès aux soins buccodentaires (1).
Santé bucco-dentaire des Libanais âgés

Peu d'études ont été réalisées pour évaluer la santé bucco-dentaire des personnes âgées au Liban. Une étude pilote menée en 2011 sur un groupe de Libanais âgés non institutionnalisés et de niveau social défavorisé a montré un taux élevé d'édentement total (41,2\%), une mauvaise hygiène orale $(43,1 \%)$, des problèmes parodontaux et dentaires ainsi que des besoins importants en soins dentaires et prothétiques difficiles à assurer $(92,1 \%)$ (5). Une seconde étude menée par El Osta et al. auprès des Libanais âgés non institutionnalisés de Beyrouth a montré que la perception de la santé bucco-dentaire était modérée chez les sujets appartenant à un milieu social aisé, mauvaise chez les sujets de niveau social moyen, et très mauvaise chez les défavorisés (6). Ceci montre les fortes inégalités sociales en santé bucco-dentaire au Liban, qui peuvent s'expliquer par des facteurs comportementaux mais aussi par un accès aux soins plus ou moins difficile selon le niveau de revenu. Certaines personnes âgées peuvent éprouver des difficultés financières après l'arrêt de l'activité professionnelle. Le coût réel des traitements dentaires, l'absence

\begin{tabular}{|c|c|c|c|c|c|c|}
\hline \multirow[t]{2}{*}{ Organisme } & \multicolumn{6}{|c|}{ Catégories d'âge (ans) } \\
\hline & $65-69$ & 70-74 & $75-79$ & 80-84 & $>85$ & Total \\
\hline Caisse Nationale de Sécurité Sociale (CNSS) ${ }^{a}$ & 24350 & 22822 & 16889 & 10002 & 2041 & 76104 \\
\hline Coopérative du secteur public & 10332 & 4070 & 4744 & 4568 & 2848 & 26562 \\
\hline Armée et Force de Sécurité Interne & 16296 & 13174 & 5937 & 3569 & 901 & 39877 \\
\hline Assurance privée (financée par l'employeur) & 376 & 0 & 0 & 0 & 0 & 376 \\
\hline Assurance privée (financée par l'assuré) & 6223 & 5576 & 3369 & 1685 & 259 & 17112 \\
\hline Municipalité et Mutuelle de l'étranger & 1196 & 1034 & 594 & 555 & 59 & 3438 \\
\hline Nombre de personnes âgées prises en charge & $\begin{array}{r}58773 \\
(48,2 \%)\end{array}$ & $\begin{array}{r}46676 \\
(44,3 \%)\end{array}$ & $\begin{array}{r}31533 \\
(44,2 \%)\end{array}$ & $\begin{array}{r}20379 \\
(44,8 \%)\end{array}$ & $\begin{array}{r}6108 \\
(33,2 \%)\end{array}$ & $\begin{array}{r}163469 \\
(45,1 \%)\end{array}$ \\
\hline Total de personnes âgées & 122014 & 105259 & 71315 & 45481 & 18371 & 362440 \\
\hline
\end{tabular}

aPersonnes âgées qui bénéficient d'une assurance facultative, ou bien personnes âgées qui bénéficient de la CNSS par le biais d'un membre de la famille. 
d'assurance couvrant les soins dentaires ou l'attitude négative des seniors à l'égard de la santé bucco-dentaire peuvent les décourager de consulter un dentiste (2).

Aucune étude d'évaluation de l'état de santé bucco-dentaire n'a été réalisée pour les Libanais âgés institutionnalisés. Par ailleurs, il règne dans les institutions libanaises une forte mentalité d'investissement et de rentabilité. De plus, le personnel médical s'intéresse à l'état de santé générale et aucun intérêt n'est accordé aux soins de la dentition des seniors. D'où l'importance de sensibiliser les médecins à l'intérêt d'une dentition saine chez la personne âgée institutionnalisée et son impact sur la santé générale, et de penser à l'intégration des dentistes dans le personnel soignant des institutions. Cela aiderait la prise en charge des problèmes bucco-dentaires des patients âgés institutionnalisés (1).

Maladies non
transmissibles et
leurs relations
avec les maladies
bucco-dentaires

Les maladies cardio-vasculaires, les affections respiratoires, le diabète et les cancers sont des maladies non transmissibles qui représentent la première cause de mortalité dans le monde et figurent parmi les principales causes de morbidité (7). Au Liban, au cours de l'année 2008, les maladies non transmissibles étaient à l'origine de $71 \%$ de tous les décès : maladies cardiovasculaires $45 \%$, diabète $2 \%$, affections respiratoires $5 \%$ et cancers $19 \%$. Ces maladies sont liées à des facteurs de risque communs qui sont le tabagisme, l'abus d'alcool, l'alimentation peu saine et la sédentarité. Le vieillissement de la population contribue à l'accroissement de l'incidence et de la prévalence des maladies non transmissibles (7).

Les maladies bucco-dentaires, qui constituent un important fardeau sanitaire, partagent les mêmes facteurs de risque des maladies non transmissibles. La consommation excessive d'alcool, le tabagisme et les mauvaises habitudes alimentaires ont un rôle important dans l'apparition des maladies non transmissibles, et peuvent également influencer négativement la santé bucco-dentaire des personnes âgées (7). Les maladies non transmissibles et bucco-dentaires constituent une menace pour l'économie du pays et peuvent accroître les inégalités dans la population. Le cercle vicieux par lequel les maladies non transmissibles et leurs facteurs de risque aggravent la pauvreté, qui elle-même contribue à l'accroissement des taux de maladies non transmissibles, crée ainsi une menace pour la santé publique et pour le développement économique et social du pays (7-9).

Ainsi, la corrélation entre ces comportements liés au mode de vie et le risque accru de caries dentaires, de maladies parodontales ou de cancers de la cavité buccale a permis d'adopter une approche intégrée de la promotion à la fois de la santé bucco-dentaire et de la santé générale. En septembre 2011, l'Organisation des Nations Unies a consacré à New York un sommet sur la prévention et la maîtrise des maladies non transmissibles en incorporant la promotion de la santé bucco-dentaire au sein de la promotion de la santé. Cette stratégie mondiale de lutte contre les maladies non transmissibles constitue une nouvelle approche pour la prévention et le contrôle des maladies bucco-dentaires dans le monde (7).

\section{Conclusion}

L'obtention du meilleur état de santé possible constitue l'un des droits fondamentaux de tout Libanais âgé quelle que soit sa situation économique ou sociale. La santé au Liban relèved'une responsabilité collective et implique un investissement social $(6,10)$. La transition démographique annoncée pour les années à venir pose un problème de santé publique et implique que les besoins en santé soient définis et anticipés. Le gouvernement ne peut y faire face qu'en prenant des mesures sanitaires et sociales appropriées pour instaurer un environnement physique et social convenable, faciliter les choix des citoyens en faveur de la santé et réduire les inégalités de santé $(6,10)$. Dans ce domaine, la prévention des maladies non transmissibles très corrélées aux maladies bucco-dentaires constitue un défi et implique d'associer tous les secteurs de la société pour trouver des moyens efficaces afin d'assurer la prévention etla maitrise de ces maladies. Les efforts de sensibilisation visant à limiter l'épidémie de maladies non transmissibles doivent être intensifiés au Liban ; le ministère de la Santé doit élaborer des politiques et des plans d'action nationaux de lutte contre les maladies non transmissibles afin de réduire non seulement la mortalité due à ces maladies, mais aussi la morbidité des maladies bucco-dentaires. Cette stratégie de prévention entrainera non seulement une augmentation du nombre de personnes qui vieillissent en bonne santé générale et bucco-dentaire mais évitera aussi de lourdes dépenses de santé chez les personnes âgées, même en l'absence d'un programme de prise en charge sanitaire pour les personnes âgées.

Conflit d'intérêt : aucun

Soutien financier : aucun. 


\section{Références}

1. El Osta N, Tubert-Jeannin S, Bou Abboud Naaman N, Hennequin M, El Osta L, Geahchan N. Vieillissement de la population Libanaise : démographie, évaluation sanitaire et impact en santé orale. Int Arab J Dent. 2011;2:5-12.

2. The World Oral Health Report: Continuous improvement of oral health in the 21st century - the approach of the WHO Global Oral Health programme. Geneva: World Health Organization; 2003.

3. Veryune JL, Lassauzay C, Peyron MA, Hennequin M. Effets du vieillissement sur les structures et les fonctions orales. Revue Geriatr. 2004;29:51-60.

4. Part three. What is the relationship between oral health and general health and well-being? Oral health in America: a report of the Surgeon General. Rockville (MD): U.S. Department of Health and Human Services, National Institute of Dental and Craniofacial Research, National Institutes of Health; $2000: 95-132$.

5. Farhat Mchayleh N, El Osta N, Tayara Sleiman R, Tannous J, Tohmé H, Boulos P. Statut bucco-dentaire au sein d'une population gériatrique libanaise. Int Arab J Dent. 2011;2:60-5.

6. El Osta N, Tubert-Jeannin S, Hennequin M, Bou Abboud Naaman N, El Osta L, Geahchan N. Comparison of the OHIP14 and GOHAl as measures of oral health among elderly in Lebanon. Health Qual Life Outcomes. 2012;10:131. PMID:23110518

7. Résolution $\mathrm{A} / 66 / 2$. Déclaration politique de la Réunion de haut niveau de l'Assemblée générale sur la prévention et la maîtrise des maladies non transmissibles. Assemblée générale des Nations Unies, soixante-sixième session, point 117 de l'ordre du jour, 19 septembre 2011 (A/RES/66/2). (http://www.un.org/en/ga/search/view_doc.asp?symbol=A/ RES/66/2\&Lang=F, consulté le 16 avril 2015).

8. Organisation mondiale de la Santé. Maladies non transmissibles : profils des pays 2011. (http://www.who.int/ nmh/countries/2011/fr/, consulté le 16 avril 2015).

9. Maladies non transmissibles. Document présenté à la $64^{\mathrm{e}}$ session du Comité régional de l'Organisation mondiale de la Santé pour le Pacifique occidental, octobre 2013 (http://www. wpro.who.int/about/regional_committee/64/documents/ wpr_rc064_08_ncd_2013_fr.pdf?ua=1, consulté le 10 mai 2015).

10. El Osta N, Tubert-Jeannin S, Hennequin M, Bou Abboud Naaman N, El Osta L, Geahchan N. Comparison of the OHIP14 and GOHAl as measures of oral health among elderly in Lebanon. Health Qual Life Outcomes. 2012;10:131. PMID:23110518 